\title{
A la conquête d'un statut professionnel : les enseignants de français en Angleterre et leurs associations (1880-1914)
}

Adèle Thomas

\section{OpenEdition}

\section{Journals}

Édition électronique

URL : https://journals.openedition.org/dhfles/1784

DOI : $10.4000 /$ dhfles. 1784

ISSN : 2221-4038

Éditeur

Société Internationale pour l'Histoire du Français Langue Étrangère ou Seconde

Édition imprimée

Date de publication : 1 juin 2005

Pagination : 214-226

ISSN : 0992-7654

Référence électronique

Adèle Thomas, «A la conquête d'un statut professionnel : les enseignants de français en Angleterre et leurs associations (1880-1914) ", Documents pour l'histoire du français langue étrangère ou seconde [En ligne], 33/34 | 2005, mis en ligne le 01 janvier 2012, consulté le 27 mai 2021. URL : http:// journals.openedition.org/dhfles/1784 ; DOI : https://doi.org/10.4000/dhfles.1784

Ce document a été généré automatiquement le 27 mai 2021.

(c) SIHFLES 


\title{
A la conquête d'un statut professionnel : les enseignants de français en Angleterre et leurs associations (1880-1914)
}

\author{
Adèle Thomas
}

1 Dans un article publié dans la Revue de Paris en 1907, Anatole Le Braz se penche sur les années d'exil de Chateaubriand en Angleterre (1793-1800) et tente de dégager la vérité " des voiles somptueux de la fiction ». Il conclut de ses recherches que si les Mémoires d'outre-tombe mettent en scène un homme qui se résigne aux pires misères de l'exil, c'est parce que l'auteur était « résolu d'avance à ne confesser jamais qu'il avait été, sinon précepteur, du moins professeur de français dans une institution privée ${ }^{1}$. Il est vrai que la piètre condition des maîtres de langues en Angleterre n'est alors pas en mesure de susciter de grands élans de fierté individuelle ou collective...

\section{Le « marchand de participes »}

On ne saurait traiter du statut des enseignants de français en Angleterre sans évoquer celui de la langue française outre-Manche. L'enseignant de français est une figure bien connue du public anglais au XIX ${ }^{\mathrm{e}}$ siècle car les catégories privilégiées du pays cultivent la langue française depuis la conquête normande. Mais s'il constitue un indice de classe doté d'un certain prestige, le français n'en demeure pas moins un simple «art d'agrément ". Son apprentissage, de rigueur pour les filles, se déroule dans un cadre privé : c'est l'affaire de précepteurs, gouvernantes et autres maîtres de langues ${ }^{2}$. Le français n'a, en revanche, pas droit de cité dans les prestigieuses public schools que fréquentent les garçons car le règne des langues anciennes y est sans partage. Il ne peut être question de les remplacer par les langues vivantes car l'on estime qu'il faudrait alors avoir recours à des étrangers pour les enseigner. Or une telle éventualité ne va 
pas sans provoquer de vives réactions. Le principal de Marlborough aurait ainsi déclaré :

Un étranger n'entrera pas dans notre esprit, dans nos mœurs, dans nos préjugés même, qui sont une part de notre nationalité. Vous ne pouvez songer à livrer à des étrangers l'œuvre générale de l'éducation ${ }^{3}$.

Depuis longtemps, ce sont effectivement des étrangers qui enseignent les langues vivantes en Angleterre. A partir du XVI ${ }^{e}$ siècle, se succèdent dans le futur Hexagone nombre d'événements politiques et religieux qui poussent certains Français à l'exil... et à la reconversion professionnelle à leur arrivée en Angleterre. Ces exilés n'enseignent pas le français par vocation mais par nécessité. L'activité, exercée dans des conditions souvent difficiles, s'avère pourtant bien peu lucrative. Aux yeux des Anglais, l'enseignement de la langue française apparaît donc souvent comme la « ressource de l'infortune et de la misère $»^{4}$. A cet égard, l'exil français présente un apport à double tranchant : il fournit à l'Angleterre des enseignants au fil des siècles, et permet ainsi d'y entretenir voire même d'y améliorer l'apprentissage du français ${ }^{5}$; mais, comme ces exilés sont parfois engagés par compassion (et même par solidarité de classe dans le cas de l'aristocratie), la langue française, tout comme l'enseignant, s'en trouvent souvent discrédités.

Par ailleurs, il ne faut pas sous-estimer la méfiance qu'éprouvent certains Anglais à l'égard de la moralité des «papistes ». Cette peur, conjuguée à celle du prosélytisme, engage ainsi nombre de familles et de directeurs d'institutions à remplacer les catholiques français par des exilés du continent protestants qui, eux aussi, se font maîtres de langues outre-Manche ${ }^{6}$.

Enfin, l'image de l'enseignant de français se caractérise par son ambivalence: si la présence d'un Français dans une école permet d'en rehausser le cachet, celui-ci doit très souvent affronter les moqueries de ses élèves et le mépris ouvertement affiché par ses collègues anglais ${ }^{7}$.

\section{2. « L'éducation étant une marchandise, c'est l'acheteur qui en détermine le choix et la quantité ${ }^{8}$}

6 Les enseignants de français ne sont pas les seuls à faire l'objet de récriminations au XIX ${ }^{e}$ siècle : une bonne partie du corps enseignant en Angleterre est alors critiquée pour son insuffisance. Il faut dire que chacun y est libre d'offrir ses services en matière d'enseignement, comme le soulignent MM. Demogeot et Montucci dans le rapport sur l'enseignement secondaire en Angleterre qu'ils adressent à Victor Duruy, ministre français de l'Instruction Publique, en 1868 :

La loi n'exige de ceux qui l'exercent aucune garantie d'aptitude ni de moralité, et la seule barrière à cette liberté illimitée, si admirée par les esprits superficiels, c'est l'opinion publique, trop longtemps tenue dans l'ignorance la plus complète des maux engendrés par l'inaptitude, ou peut-être même par la mauvaise foi des entrepreneurs d'éducation'.

7 En Angleterre, le personnel enseignant n'est pas recruté en vertu de compétences intellectuelles certifiées par des examens comme en France. On n'y recherche d'ailleurs pas tant un spécialiste qu'une personne capable de transmettre des valeurs. L'étendue de ses connaissances n'est donc pas primordiale. L'aristocratie tend à se fier aux recommandations de la famille ou des amis ; la bourgeoisie fait plutôt appel aux petites 
annonces et aux agences de placement. ${ }^{10}$ Aucun corps de contrôle n'est mis en place pour vérifier les aptitudes de tous ces enseignants.

Signalons que les exigences de ces employeurs s'avèrent souvent peu réalistes: le précepteur, la gouvernante doivent ainsi enseigner bon nombre de disciplines différentes. Dans son ouvrage intitulé Drat the Boys! le Français Léon-Paul Blouët nous fait partager sa première expérience d'enseignement en Angleterre. Engagé en qualité de resident master dans une école du Somersetshire dirigée par un certain Mr R., il découvre qu'il y est le seul enseignant et qu'il doit donc se rendre utile. Il avoue enseigner plus de disciplines que ses compétences ne devraient le permettre, mais note qu'il donne quand même satisfaction. Un mois après son arrivée, il écrit dans son journal, non sans humour :

23 août 1873. Mr R. me suggère d'enseigner deux ou trois nouvelles matières. Je suis prêt à me conformer à ses désirs, mais je refuse catégoriquement d'enseigner la valse à trois temps ${ }^{11}$.

Le mode de recrutement des gouvernantes, amenées elles aussi à enseigner le français, se révèle symptomatique de l'attente des familles en matière d'éducation. Bien souvent, celles-ci s'enquièrent du statut social et de la pratique religieuse d'une gouvernante avant même de se préoccuper de ses capacités intellectuelles. Une bonne gouvernante est avant tout une lady. Or il est rare qu'une jeune femme issue de la bonne société anglaise devienne gouvernante par choix : une telle activité constitue plutôt le dernier recours de celle qui doit subvenir à ses besoins dès lors que sa famille n'est plus en mesure de le faire. L'étrangère, quant à elle, fait figure de professionnelle : les Anglais apprécient sa formation académique et pédagogique... a fortiori si elle est protestante. Cependant, le prestige lié à l'emploi d'une Française permet parfois de dépasser les réticences que suscite sa religion lorsque celle-ci est catholique ${ }^{12}$.

\section{Essor du mouvement associatif et professionnalisation}

L'intérêt des Anglais pour l'éducation, et notamment pour la question des langues vivantes, va croissant à la fin du XIX siècle. Il semble que l'introduction progressive de cette discipline dans les écoles soit d'ailleurs une concession faite à l'opinion publique, convaincue de l'utilité d'un tel apprentissage ${ }^{13}$.

11 Pourtant, certains Français jugent la situation critique : après le traumatisme de la défaite de 1870, il leur paraît insupportable de se voir évincer au profit de protestants dont le français n'est pas la langue première... à plus forte raison lorsque ceux-ci sont de nationalité allemande ${ }^{14}$. A leurs yeux, l'exclusion progressive des Français ne peut d'ailleurs que ternir l'image, déjà peu flatteuse, de l'enseignant de français. Ils décident donc de se regrouper et créent en 1881 la Société Nationale des Professeurs de français en Angleterre ${ }^{15}$.

12 Il ne s'agit pas là d'une initiative isolée : nombre d'enseignants se regroupent alors en Angleterre dans l'espoir de voir leur activité reconnue ${ }^{16}$. La SNPF s'inscrit donc dans ce courant de professionnalisation qui repose, non pas sur l'Université et la création d'examens comme en France, mais sur l'essor du mouvement associatif. Le mode de sélection des enseignants tel qu'il se pratique sur le continent ne traverse pas la Manche: soucieuse de la liberté de l'enseignement, la société anglaise oppose 
également une certaine résistance à la mobilité sociale que pourraient permettre tous ces examens et autres concours ${ }^{17}$.

\section{Le rassemblement d'une élite : ambitions de la SNPF}

La SNPF est une association sélective. Par souci d'honorabilité, elle recrute ses membres sur des critères très stricts. Les Sociétaires estiment en effet que «la profession est envahie par beaucoup de gens des deux sexes, qui, non contents d'usurper la qualité de Français, s'attribuent des aptitudes et des connaissances qu'ils n'ont pas. » Il s'agit donc pour eux de filtrer la profession, de mettre en place un « cordon sanitaire ». Le critère de nationalité s'avère essentiel à leurs yeux: nul autre qu'un Français ne saurait enseigner la langue française car il faut avant tout posséder «le tempérament de la nation $»^{18}$. Tout comme dans les syndicats et clubs anglais, chaque candidat doit être présenté par deux membres ${ }^{19}$. Les Sociétaires souhaitent ainsi se substituer favorablement aux agents de placement et démontrer aux Anglais qu'il est désormais possible de trouver des enseignants de français compétents.

Les Sociétaires fondateurs appartiennent eux-mêmes à une élite enseignante. Après avoir, dans l'ensemble, connu des débuts difficiles ${ }^{20}$, ils occupent ces quelques postes confortables qui existent tout de même dans certaines universités et public schools ${ }^{21}$. Ce sont des gens qui connaissent une certaine réussite et qui ne souffrent plus, lorsqu'ils créent la SNPF, de cette misère qui affecte tant d'enseignants français. Ils se caractérisent par leur engagement dans la vie politique et associative, avant et après leur exil de France. Plusieurs générations se côtoient ainsi au sein de la SNPF : Charles Cassal est un ancien élu de la période des Cent Jours en France ${ }^{22}$; Alfred Hamonet est « un homme de $48 »^{23}$; Albert Barrère, frère du futur ambassadeur à Rome, a pris part aux événements de la Commune. En Angleterre, ces hommes devenus enseignants conservent souvent un lien avec la France: Georges Petilleau représente la Société des Gens de Lettres en Angleterre, tandis que d'autres se font correspondants pour la presse française, ou écrivent des ouvrages sur l'Angleterre à l'intention des Français.

Leur ambition est de représenter «la France intellectuelle en Angleterre $»^{24}$, et leur action vise à satisfaire un double objectif: s'attaquer aux préjugés qui entachent la réputation de leur activité, mais aussi relever le statut de la langue française. Ils œuvrent ainsi en faveur d'une plus large diffusion du français dans le système scolaire et suggèrent nombre de réformes afin que celui-ci ne soit plus enseigné comme une langue morte.

Pour se donner plus de poids mais aussi plus d'éclat, la Société recherche l'appui de personnalités anglaises et françaises. Le comité d'honneur français, présidé par Victor Hugo, se constitue notamment de Louis Blanc, Alexandre Dumas, Léon Gambetta, Jean Macé, Ernest Renan ${ }^{25}$. Grâce à ses congrès et à son journal, le Français, la SNPF fait connaître son action au grand public tout autant qu'elle développe un certain esprit de corps chez les enseignants. 


\section{Une action reconnue et encouragée}

17 La croissance de l'association, qui se compose de 87 membres en 1881 (les Sociétaires fondateurs), de 145 sociétaires en $1887^{26}$, puis de 193 membres en $1914^{27}$, peut être perçue comme la preuve de sa popularité et d'un certain crédit.

Mais si l'on dépasse cette approche quantitative, l'on peut constater que la SNPF éveille également l'intérêt des Français de l'Hexagone en des temps qui voient naître l'Alliance française (1883). Les lettres du comité d'honneur adressées à la SNPF lors de ses congrès s'avèrent ainsi particulièrement éloquentes : le marchand de participes n'est plus; il laisse place au missionnaire laïc, qui contribue au rayonnement de la France par la diffusion de sa langue et de sa culture à l'étranger. Si les crédits et autres subventions ne sont pas toujours au rendez-vous, la SNPF bénéficie cependant du soutien des ambassadeurs en poste à Londres, ${ }^{28}$ qui perçoivent l'intérêt que peut avoir une telle action pour la France après le revers de 1870.

L'association est également reconnue dans les milieux anglais : Cambridge et Oxford, bastions des études classiques, accueillent ses congrès en 1887 et 1888 ; les réceptions du Lord-maire de Londres ${ }^{29}$ constituent autant d'occasions de féliciter la SNPF pour les progrès effectués. Enfin, les activités de l'association trouvent un certain écho dans la presse anglaise, et notamment dans le Times. Ce journal consacre ainsi tout un éditorial à la condition des enseignants français en Angleterre lors du $3^{\text {ème }}$ congrès de la SNPF : on y rappelle au grand public que les Français qui entreprennent d'enseigner leur langue en Angleterre doivent faire face à de nombreux obstacles et préjugés... et qu'ils ne sont donc pas seuls responsables des médiocres résultats obtenus ${ }^{30}$.

Certains Français souhaitent, quant à eux, que la SNPF aille encore plus loin dans son entreprise de sélection des enseignants. A l'occasion du congrès de 1886, le Dr. Beljame, délégué du Ministre de l'Instruction Publique, déclare ainsi :

Vous êtes solidement constitués; vous comptez parmi vos membres des hommes qui font justement autorité dans l'enseignement; vous possédez, en un mot, tous les éléments d'un jury d'examen ${ }^{31}$.

Convaincu des bienfaits qu'ont apportés les examens dans l'enseignement des langues en France, il suggère donc à la SNPF de prendre une initiative similaire, puisqu'il n'est pas permis, selon lui, d'espérer que le gouvernement anglais s'en charge.

En 1919, le rapport Marchat sur les œuvres françaises à Londres, mené par le consulat général de France, suggère même que la SNPF prenne part à un « contrôle technique du corps professoral », qui serait mis en place avec le concours du Ministère du Travail à Londres afin de remédier à l'insuffisance des institutrices ${ }^{32}$.

\section{Qui doit enseigner les langues vivantes ?}

Cette évocation de l'insuffisance des institutrices nous amène à considérer l'une des limites de l'action de la SNPF. Si les hommes profitent de cette professionnalisation de l'enseignement $d u$ français, il semble en revanche que le statut des femmes ne soit guère plus enviable : ces dernières font même l'objet d'attaques masculines répétées. En 1894, le Courrier de Londres, organe de la colonie française, fait ainsi paraitre dans ses colonnes des propos très virulents à leur encontre : 
[...] nous osons dire que, même dans de très grandes familles, la soi-disant institutrice française qui possède un appartement indépendant et une femme de chambre à ses ordres, serait parfois mieux à sa place dans les sous-sols, aux côtés de la cuisinière, avec laquelle elle pourrait avantageusement lutter en dévergondage orthographique $^{33}$. compétence, alors asservis par la SNPF à cette notion de nationalité. La revue pédagogique, Journal of Education, accuse ainsi les Sociétaires d'avoir fait preuve d'un chauvinisme exagéré lors du congrès de 1882: "Ils ont eu raison de dénoncer le charlatanisme, mais tous les Allemands, Polonais et Suisses qui enseignent le français en Angleterre ne sont pas des charlatans ou des intrus $»^{38}$. 

thème : «Qui doit enseigner les langues vivantes ? ", où sont exposés cette fois tous les défauts de l'enseignant français. Non seulement le Français ferait l'objet d'un mépris inné chez les jeunes Anglais en raison de l'insularité de leur pays, ${ }^{39}$ mais le Français serait aussi inférieur à ses collègues anglais ${ }^{40} . .$. tant et si bien que l'on finit par poser la question de la valeur éducative de l'apprentissage de la prononciation, au vu des risques encourus. L'intérêt d'une bonne prononciation mérite-t-il vraiment que l'on engage un Français, quand le passé de celui-ci peut s'avérer obscur, son anglais approximatif, et son maintien de la discipline quasi-nul ? ${ }^{41}$

Notons que tout ce discours d'hostilité envers les professeurs étrangers ne se manifeste que dès lors qu'il s'agit de l'éducation des garçons, au sein de l'institution scolaire. Il n'est ici jamais question des risques encourus par les filles, qui apprennent le français dans un cadre plus familial. L'on peut donc se demander si l'étranger est perçu comme une menace au développement de l'esprit national chez les garçons... tout en gardant à l'esprit que des motivations plus matérielles ont pu susciter ces prises de position car les postes en question sont alors mieux payés et valorisés.

32 L'introduction progressive de l'enseignement $d u$ français dans les écoles et les universités fait donc naître le désir de voir l'étranger remplacé par un Anglais. Le Sociétaire Alfred Hamonet se plaint d'ailleurs de la « croisade » alors menée contre les enseignants étrangers ${ }^{42}$. Rappelons que ce type de réaction corporatiste s'observe également en France; l'Angleterre estime d'ailleurs suivre en cela l'exemple de la France et de l'Allemagne ${ }^{43}$.

33 En 1892, les enseignants anglais de langues vivantes créent la Modern Language Association (MLA) qui, d'après Harry Radford, n'aura de cesse de critiquer le recrutement d'enseignants étrangers ${ }^{44}$. Leur position trouve d'ailleurs un certain écho puisqu'en 1918, la conclusion $n^{\circ} 38$ du rapport sur l'enseignement des langues vivantes en Grande-Bretagne spécifie que la direction de ces études dans les universités devrait être confiée à des Britanniques ${ }^{45}$.

Voici donc tracée à grands traits l'évolution du maître de français outre-Manche. En l'espace de quelques décennies, l'isolement des enseignants français laisse place à une impérieuse volonté de rassemblement. Le traumatisme de 1870 invite ainsi certains Français à l'action: il s'agit pour eux de voir le «marchand de participes » enfin remplacé par une figure plus respectable... et respectée. Une association telle que la SNPF se lance donc dans une entreprise de sélection des enseignants : elle définit, à cet effet, des critères d'admission et pose ainsi la question des aptitudes et compétences requises pour l'enseignement du français. Or ces critères de sélection sont tels qu'ils ne peuvent qu'engager un débat fort nourri : Qui doit enseigner les langues vivantes en Angleterre ? Des étrangers ou des Anglais? La société anglaise penche pour la seconde option au fur et à mesure que cette discipline entre dans les écoles et les universités et que cet enseignement gagne en éclat grâce à l'essor et aux efforts de diverses associations. Mais que penser du sort des femmes, qui se voient contraintes de créer leurs propres associations afin de défendre leurs intérêts? A défaut de pouvoir symbolique, celles-ci acquièrent un pouvoir numérique assez rapide... ce qui pose encore une fois la question du statut des enseignants de français, quand on sait que la féminisation d'une activité est souvent associée à une perte de prestige de cette dernière. 


\title{
BIBLIOGRAPHIE
}

\section{Bibliographie}

\section{Sources primaires}

\author{
Courrier de Londres \\ Le Français, bulletin de la SNPF \\ Journal of Education \\ Modern Language Journal \\ The Times
}

DEMOGEOT, J. et MONTUCCI, H. (1868), De l'enseignement secondaire en Angleterre et en Ecosse, rapport adressé à son Exc. M. le Ministre de l'Instruction Publique, Paris, Imprimerie Impériale, $664 \mathrm{p}$.

HAMONET, Alfred (1882), Réflexions sur l'enseignement du français en Angleterre, texte du discours prononcé à Londres le 13 janvier 1882 à l'occasion du Congrès des professeurs de langue française (12 au 14/01/1882), Londres, $18 \mathrm{p}$.

LEATHES, Stanley Mordaunt - Great Britain Board of Education - Committee on the Position of Modern Languages in the Educational System of Great Britain (1918). Report of the Committee appointed by the Prime Minister to enquire into the Position of Modern Languages in the Educational System of Great Britain, Londres, His Majesty's Stationery Office, $82 \mathrm{p}$.

MARCHAT (1919), « Euvres françaises et d'influence française de la circonscription du consulat général de France à Londres », Auteur : Marchat, 22/04/-06/05/1919, Dossier « GrandeBretagne ", Fonds Services des Euvres Françaises à l'Etranger, n¹41, Archives du Ministère des Affaires Etrangères

O'RELL, Max (1886), Drat the Boys! Or Recollections of an ex-French master in England, Londres, Field and Tuer, $170 \mathrm{p}$.

SNPFA, Congrès de la Société Nationale des Professeurs de français en Angleterre - Quatrième session, tenue à Londres les 2 et 3 janvier 1885, Londres, Hachette, 118 p.

SNPFA, Congrès de la Société Nationale des Professeurs de français en Angleterre - Cinquième session, tenue à Londres les 8 et 9 janvier 1886, Londres, Hachette, 89 p.

SNPFA, Congrès de la Société Nationale des Professeurs de français en Angleterre - Sixième session, tenue à Londres le 12 janvier 1887 et à Oxford « Balliol College » le 13 janvier 1887, Londres, Hachette, 105 p.

SNPFA (1932), La Journée du Cinquantenaire de la Société des Professeurs de français en Angleterre, tenue sous la présidence de Son Excellence, M. de Fleuriau, Ambassadeur de France en Grande-Bretagne, le samedi 31 octobre 1931, Pornic, La Vague, 76 p.

SNPFA, Statuts et règlement, votés en AG à Londres le 12/01/1882

SPLFA (1832), Introduction aux Annuaires de la Société des Professeurs de Langue Française en Angleterre, Londres, Dulau, 259 p. 


\section{Sources secondaires}

ARMYTAGE, W. H. G. (1970, $1^{\text {ère }}$ éd. 1964), Four Hundred Years of English Education, Cambridge, CUP, $353 \mathrm{p}$.

BAYLEY, S. N. et RONISH, D. Y. (1992), « Gender, Modern Languages and the Curriculum in Victorian England », History of Education, vol. 21, n 4, 363-382.

BAYLEY, Susan (1998), « The Direct Method and Modern Language Teaching in England 1880-1918 », History of Education, vol. 27, n 1, 39-57.

BERGEN, Barry H. (1982), « Only a Schoolmaster : Gender, Class, and the Effort to Professionalize Elementary Teaching in England, 1870-1910 », History of Education Quarterly, vol. 22, n 1, 1-21.

BRUNOT, Ferdinand (1967, 1 ère éd. à partir de 1905), Histoire de la langue française, t. V, VIII et XI, Paris, A. Colin.

GERBOD, Paul (1988), «L'Enseignement de la langue française en Grande-Bretagne au XIXe siècle (1800-1870) », Documents pour l'histoire du français langue étrangère ou seconde, $\mathrm{n}^{\circ} 2,8-11$

GERBOD, Paul (1991), « L'Enseignement de la langue française en Angleterre au XIX ${ }^{\mathrm{e}}$ siècle (1800-1860) », L'Information historique, vol. 53, n 5, 180-186

HAWKINS, Eric (1987, $1^{\text {ère }}$ éd. 1981), Modern Languages in the Curriculum, Cambridge, Cambridge University Press, $322 \mathrm{p}$.

HOLCOMBE, Lee (1973), Victorian Ladies at Work, Newton Abbot, David \& Charles, 253 p

HUGHES, Kathryn (2001, $1^{\text {ère }}$ éd. 1993), The Victorian Governess, Londres, Hambledon and London, $256 \mathrm{p}$.

JACKSON, John Archer (1970), Professions and Professionalization, Cambridge, CUP, 226p

KIBBEE, Douglas A. (1991), For to Speke Frenche Trewly: the French Language in England, 1000-1600 : its Status, Description and Instruction, Amsterdam/Philadelphie, J. Benjamins, 268 p.

LAMBLEY, Kathleen (1920), The Teaching and Cultivation of the French Language in England during Tudor and Stuart Times, with an introductory chapter on the preceding period, Manchester, the University Press, $438 \mathrm{p}$.

LAWSON, John et SILVER, Harold (1973), A Social History of Education in England, Londres, Methuen, $502 \mathrm{p}$.

LE BRAZ, Anatole (1907), « Chateaubriand professeur de français », Revue de Paris, livraison du 15 août 1907, 791-808.

PETERSON, M. Jeanne (1972), « The Victorian Governess: Status Incongruence in Family and Society ", in Vicinus, Martha (dir.) Suffer and Be Still, Bloomington \& Londres, Indiana University Press, $239 \mathrm{p}$.

RADFORD, H. (1985), « Modern Languages and the Curriculum in English Secondary Schools ", in Goodson, I. (dir.) Social Histories of the Secondary Curriculum, Londres, Falmer Press, 203-237.

READER, W. J. (1966), Professional Men: the Rise of the Professional Classes in $19^{\text {th }}$ Century England, Londres, Weidenfeld \& Nicolson, 248 p. 


\section{NOTES}

1. A. Le Braz $(1907: 791,808)$

2. S. N. Bayley et D. Y. Ronish (1992:367-369) ; P. Gerbod (1988:9-10)

3. J. Demogeot et H. Montucci (1868: 72)

4. SPLFA (1832:38)

5. K. Lambley $(1920: 57)$

6. F. Brunot (éd. 1967 : VIII, 264) ; F. Baldensperger dans F. Brunot (éd. 1967 : XI, 192)

7. S. N. Bayley et D. Y. Ronish (1992: 366) ; H. Radford (1985: 209)

8. J. Demogeot et H. Montucci (1868: 304)

9. J. Demogeot et H. Montucci $(1868: 303)$

10. K. Hughes (éd. 2001: 43) ; J. Demogeot et H. Montucci (1868 : 303-304)

11. Max O'Rell (1886: 38-41) : «23rd August, 1873. Mr R. proposes that I should teach two or three new subjects. I am ready to comply with his wishes; but I sternly refuse to teach la valse à trois temps. "

12. K. Hughes (éd. $2001: 48-49,107$ )

13. E. Hawkins (éd. $1987: 117-118$ ) ; J. Demogeot et H. Montucci (1868: 74)

14. A. Hamonet (Discours, $1882: 14$ ) : «Au surplus, nous sommes en état de légitime défense ; il nous est bien permis de refuser à l'Allemand l'accès de notre domaine, quand nous voyons $M$. de Bismarck porter la main, une main de fer! sur la langue française et en interdire l'emploi en Alsace et en Lorraine ».

15. A noter : les Français avaient déjà tenté de s'associer à deux reprises au cours du XIXe siècle, mais sans grand succès.

16. The Times (31/10/1900) ; B. H. Bergen (1982) ; L. Holcombe (1973 : 34-66).

17. The Times (18/01/1877 et 05/06/1899) ; B. H. Bergen (1982) ; K. Hughes (éd. 2001: 40, 188)

18. A. Hamonet (Discours, $1882: 15,13,7$ )

19. SNPFA, Statuts et règlement, (1882: 7), Ch. V : «Admission, Droits et Devoirs des Sociétaires », article 87: "Tout Français, enseignant le français en Angleterre depuis au moins un an, ou y ayant enseigné pendant au moins cinq ans, peut être admis à faire partie de la Société - sur la présentation écrite de deux membres, qui certifieront la moralité et l'aptitude du candidat. »

20. SNPFA (Congrès, $1885: 54$ ). Georges Petilleau, après avoir décrit le quotidien difficile des professeurs français, notamment dans les private schools, déclare ainsi : «c'est par milliers que se nombrent ces Private Schools où tant de vigoureux talents s'étiolent dans le struggle for life! Oh! Ne protestez pas, Messieurs, par amour-propre. A part de rares exceptions, vous avez passé par là, avant d'appartenir à des écoles sérieuses. J’y ai passé, moi aussi ! »

21. SNPFA (Congrès, $1885: 35$ )

Jules Bué enseigne à l'université d'Oxford; Georges Petilleau est professeur de français à Charterhouse ; Charles Cassal est professeur d'université à Londres.

22. SNPFA (Congrès, $1885: 35$ )

23. SNPFA (Journée du cinquantenaire, $1932: 12$ )

24. Le Français $\left(1889, \mathrm{n}^{\circ} 75: 26\right)$

25. A. Hamonet (Discours, $1882: 1$ )

26. SNPFA (Congrès, $1887: 11-12$ )

27. Le Français $\left(1914, \mathrm{n}^{\circ} 199\right.$ : 63-68)

28. W. H. Waddington; Paul Cambon

29. Réceptions organisées à l'occasion de la distribution des prix du Grand Concours de Langue et de Littérature françaises établi par la SNPF

30. The Times (04/01/1884: « The position of a French teacher in England »)

31. SNPFA, (Congrès de $1886: 81-82$ ) 
32. Marchat (1919: 55-57) : «En raison de la législation anglaise actuelle, il semble qu'un tel résultat pourrait être facilement obtenu, tout au moins en ce qui concerne celles de nos compatriotes qui ne sont pas encore en Angleterre. Il suffirait, en effet, de s'entendre avec le Ministry of Labour, dont l'autorisation est requise, pour que ce Département soumette, au préalable, les demandes dont il serait saisi, à la sanction de l' 'Institut', de la 'Société des Professeurs', et surtout du 'Home' ».

33. Courrier de Londres (11/07/1886, dans la série d'articles «l'éducation en Angleterre - les garçons »)

34. Journal of Education (1894: 681)

35. Le Français $\left(1884, \mathrm{n}^{\circ} 32: 139\right)$

36. Le Français $\left(1883, \mathrm{n}^{\circ} 13: 71\right)$

37. Lettre de Pierre Foncin, 24/01/1899, Fonds Ambassade de France en Grande-Bretagne, Série Chancellerie, $\mathrm{n}^{\circ}$ 267, Archives du Ministère des Affaires Etrangères.

38. Journal of Education (1882: 39). "They were right in denouncing quackery; but all Germans, Poles, and Swiss, who teach French in England, are not quacks or interlopers. »

39. Journal of Education (1882: 87), lettre de J.M. Horsburgh, Radley College

40. Journal of Education (1892: 136)

41. Journal of Education (1892:120)

42. Journal of Education (1894:338-339)

43. Journal of Education (1882: 87)

44. H. Radford (1985 : 218); Modern Language Journal (1934, vol. 19, $\mathrm{n}^{\circ} 2$ : 140-144), Statement of policy : «It is a fundamental part of the policy of the MLA that the teaching of modern languages in this country should be mainly in the hands of men and women of British nationality ».

45. Stanley Mordaunt Leathes (1918: 63)

\section{RÉSUMÉS}

À la fin du XIX $\mathrm{X}^{\mathrm{e}}$ siècle, nombre d'exilés français devenus maîtres de langue en Angleterre se soucient de la précarité de leur situation. Les Anglais tendent en effet à préférer, pour l'enseignement $d u$ français, les protestants du continent aux Français catholiques. Ces Français s'inquiètent donc, non seulement de leur exclusion progressive, mais aussi des conséquences que celle-ci peut avoir sur l'image, déjà peu flatteuse, des enseignants de français. Par leur association, qui recrute ses membres sur des critères de nationalité, de compétence et de moralité très stricts, ils espèrent démontrer aux Anglais qu'ils ne sont pas de simples «marchands de participes »... interchangeables. Mais celles et ceux qui enseignent le français en Angleterre ne se retrouvent pas tous dans ce premier discours. Les institutrices françaises et les Anglais créent donc leurs propres associations, afin de défendre leurs positions.

At the end of the $19^{\text {th }}$ century, many of the French exiles who took up language teaching as an occupation in England feel concerned about the insecurity of their position: when recruiting teachers of French, employers tend to prefer continental Protestants to French Catholics. These teachers of French nationality therefore worry about an exclusion which, according to them, is not to help their profession gain some lustre. They decide to form an association whose members are to be selected upon strict national, academic and moral criteria and hope thereby to convince English people that they are much more than easily replaceable "marchands de participes". But a 
number of teachers of French feel rejected by such an exclusive attitude. Who is to teach languages then? Women and English-born teachers from their own associations and join the debate...

INDEX

Mots-clés : Angleterre, Catholiques, institutrices, Maître de langues, professeur de français, Protestants Société Nationale des Professeurs de français en Angleterre (SNPFA), statut professionnel, XIXe siècle

Keywords : Catholics, England, Language schoolmaster, professionnal status, Protestants, Société Nationale des Professeurs de français en Angleterre (SNPFA), teacher of French, women teachers, XIXth century

\section{AUTEUR}

\section{ADĖLE THOMAS}

Université Paris XIII 